\title{
The orthosymplectic Lie supergroup in harmonic analysis
}

\author{
Kevin Coulembier \\ Department of Mathematical Analysis, Faculty of Engineering, Ghent University, Belgium
}

\begin{abstract}
The study of harmonic analysis in superspace led to the Howe dual pair $\left(O(m) \times S p(2 n), \mathfrak{s l}_{2}\right)$. This Howe dual pair is incomplete, which has several implications. These problems can be solved by introducing the orthosymplectic Lie supergroup $O S p(m \mid 2 n)$. We introduce Lie supergroups in the supermanifold setting and show that everything is captured in the Harish-Chandra pair. We show the uniqueness of the supersphere integration as an orthosymplectically invariant linear functional. Finally we study the $\mathfrak{o s p}(m \mid 2 n)$-representations of supersymmetric tensors for the natural module for osp $(m \mid 2 n)$.

Keywords: Howe dual pair, Lie supergroup, Lie superalgebra, harmonic analysis, supersphere PACS: $02.10 . \mathrm{Ud}, 20.30 . \mathrm{Cj}, 02.30 . \mathrm{Px}$
\end{abstract}

\section{PRELIMINARIES}

Superspaces are spaces where one considers not only commuting but also anti-commuting variables. The $2 n$ anticommuting variables $\grave{x_{i}}$ generate the complex Grassmann algebra $\Lambda_{2 n}$.

Definition 1. A supermanifold of dimension $m \mid 2 n$ is a ringed space $\mathscr{M}=\left(\mathscr{M}_{0}, \mathscr{O}_{\mathscr{M}}\right)$, with $\mathscr{M}_{0}$ the underlying manifold of dimension $m$ and $\mathscr{O}_{\mathscr{M}}$ the structure sheaf which is a sheaf of super-algebras with unity on $\mathscr{M}_{0}$. The sheaf $\mathscr{O} \mathscr{M}$ satisfies the local triviality condition: there exists an open cover $\left\{U_{i}\right\}_{i \in I}$ of $\mathscr{M}_{0}$ and isomorphisms of sheaves of super algebras $T_{i}: \mathscr{O}_{\mathscr{M}}^{U_{i}} \rightarrow \mathscr{C}_{U_{i}}^{\infty} \otimes \Lambda_{2 n}$.

The sections of the structure sheaf are referred to as superfunctions on $\mathscr{M}$. A morphism of supermanifolds $\Phi: \mathscr{M} \rightarrow \mathscr{N}$ is a morphism of ringed spaces $\left(\phi, \phi^{\sharp}\right), \phi: \mathscr{M}_{0} \rightarrow \mathscr{N}_{0}, \phi^{\sharp}: \mathscr{O}_{\mathscr{N}} \rightarrow \phi_{*} \mathscr{O} \mathscr{M}$. Each point $p \in \mathscr{M}_{0}$ defines a morphism $\delta_{p}:(\{*\}, \mathbb{R}) \rightarrow\left(\mathscr{M}_{0}, \mathscr{O}_{\mathscr{M}}\right)$, with $\{*\}$ the manifold consisting of one point. This is defined by $\delta_{p}^{\sharp}(f)=[f]_{0}(p)$ where $[f]_{0}$ is the canonical projection $\mathscr{O}_{\mathscr{M}} \rightarrow \mathscr{C}_{\mathscr{M}_{0}}^{\infty}$.

The flat supermanifold is given by $\mathbb{R}^{m \mid 2 n}=\left(\mathbb{R}^{m}, \mathscr{C}_{\mathbb{R}^{m}}^{\infty} \otimes \Lambda_{2 n}\right)$. The supervector $\mathbf{x}$ is defined as

$$
\mathbf{x}=\left(X_{1}, \cdots, X_{m+2 n}\right)=(\underline{x}, \underline{\grave{x}})=\left(x_{1}, \cdots, x_{m}, \grave{x_{1}}, \cdots, \grave{x_{2 n}}\right) .
$$

The orthosymplectic metric $g$ on $\mathbb{R}^{m \mid 2 n}$ is defined as $g \in \mathbb{R}^{(m+2 n) \times(m+2 n)}$

$$
g=\left(\begin{array}{c|c}
I_{m} & 0 \\
\hline 0 & J_{2 n}
\end{array}\right) \quad \text { with } J_{2 n} \in \mathbb{R}^{2 n \times 2 n} \text { given by } \quad J_{2 n}=\frac{1}{2}\left(\begin{array}{ccccc}
0 & -1 & & & \\
1 & 0 & & & \\
& & \ddots & & \\
& & 0 & -1 \\
& & 1 & 0
\end{array}\right)
$$

We define $X^{j}=X_{i} g^{i j}$. The square of the 'radial coordinate' is given by

$$
R^{2}=\langle\mathbf{x}, \mathbf{x}\rangle=\sum_{j=1}^{m+2 n} X^{j} X_{j}=\sum_{i=1}^{m} x_{i}^{2}-\sum_{j=1}^{n} \grave{x}_{2 j-1} \grave{x_{2 j}}=r^{2}+\theta^{2},
$$

see [3]. The Lie group of matrices $S \in \mathbb{R}^{(m+2 n) \times(m+2 n)}$ which satisfy $\langle S \cdot \mathbf{x}, S \cdot \mathbf{x}\rangle=\langle\mathbf{x}, \mathbf{x}\rangle$, is identified with $O(m) \times$ $\operatorname{Sp}(2 n)$, see [4].

The super gradient is defined as $\nabla=\left(\partial_{X^{1}}, \cdots, \partial_{X^{m+2 n}}\right)$. From this expression and the metric we obtain the Laplace operator

$$
\nabla^{2}=\langle\nabla, \nabla\rangle=\sum_{k=1}^{m+2 n} \partial_{X_{k}} \partial_{X^{k}}=\sum_{i=1}^{m} \partial_{x_{i}}^{2}-4 \sum_{j=1}^{n} \partial_{\grave{x}_{2 j-1}} \partial_{\grave{x}_{2 j}} .
$$


The super Euler operator is defined by $\mathbb{E}=\langle\mathbf{x}, \nabla\rangle$. The operators $-\nabla^{2} / 2, R^{2} / 2$ and $\mathbb{E}+\frac{m-2 n}{2}$, generate the $\mathfrak{s l}_{2}$ Lie algebra ([4]).

The space of super polynomials is given by

$$
\mathscr{P}=\mathbb{R}\left[x_{1}, \cdots, x_{m}\right] \otimes \Lambda_{2 n} \subset \mathscr{O}_{\mathbb{R}^{m \mid 2 n}}\left(\mathbb{R}^{m}\right)=\mathscr{C}^{\infty}\left(\mathbb{R}^{m}\right) \otimes \Lambda_{2 n}
$$

The polynomials of degree $k$ are the elements $P \in \mathscr{P}$ which satisfy $\mathbb{E} P=k P$, the corresponding spaces are denoted $\mathscr{P}_{k}$. The polynomial null-solutions of $\nabla^{2}$ of fixed degree are called spherical harmonics, the spaces are denoted $\mathscr{H}_{k}=\mathscr{P}_{k} \cap \operatorname{ker} \nabla^{2}$.

Lemma 1 (Fischer decomposition). If $m-2 n \notin-2 \mathbb{N}$, $\mathscr{P}$ decomposes as

$$
\mathscr{P}=\bigoplus_{k=0}^{\infty} \mathscr{P}_{k}=\bigoplus_{j=0}^{\infty} \bigoplus_{k=0}^{\infty} R^{2 j} \mathscr{H}_{k}
$$

Since $R^{2}, \nabla^{2}$ and $\mathbb{E}+\frac{m-2 n}{2}$, which generate $\mathfrak{s l}_{2}$, are $O(m) \times S p(2 n)$-invariant we obtain the Howe dual pair $\left(O(m) \times S p(2 n), \mathfrak{s l}_{2}\right)$. This is closely related to the Fischer decomposition (1). The blocks $\bigoplus_{j} R^{2 j} \mathscr{H}_{k}$ are irreducible $\mathfrak{s l}_{2}$ representations with weight vectors $R^{2 j} \mathscr{H}_{k}$. The weight vectors $R^{2 j} \mathscr{H}_{k}$ are $O(m) \times S p(2 n)$-representations. In order to have a complete Howe dual pair, the blocks $R^{2 j} \mathscr{H}_{k}$ should be the irreducible parts of the representation $(\mathscr{P}, O(m) \times S p(2 n))$. However in superspace these blocks are not irreducible, see [4].

Another shortcoming of the Howe dual pair is that all functions of the form $f\left(r^{2}, \theta^{2}\right)$ are invariant under $O(m) \times$ $S p(2 n)$. To have a complete Howe dual pair, only the polynomals $R^{2 j}$ should be one dimensional subrepresentations of $\mathscr{P}$.

Finally we introduce integration over the supersphere for polynomials, see [4],

Theorem 1. When $m \neq 0$, the only (up to a multiplicative constant) linear functional $T: \mathscr{P} \rightarrow \mathbb{R}$ satisfying the following properties for all $f(\mathbf{x}) \in \mathscr{P}$ :

- $T\left(R^{2} f(\mathbf{x})\right)=T(f(\mathbf{x}))$

- $T(f(S \cdot \mathbf{x}))=T(f(\mathbf{x})), \quad \forall S \in O(m) \times S p(2 n)$

$\cdot k \neq l \Longrightarrow T\left(\mathscr{H}_{k} \mathscr{H}_{l}\right)=0$

is given by the Pizzetti integral

$$
\int_{\mathbb{S}^{m-1 \mid 2 n}} f(\mathbf{x})=\sum_{k=0}^{\infty} \frac{2 \pi^{m / 2-n}}{2^{2 k} k ! \Gamma(k+m / 2-n)}\left(\nabla^{2 k} f\right)(0), \quad f(\mathbf{x}) \in \mathscr{P} .
$$

In the purely bosonic case, the third condition is implied by the first two. Summarizing, the theory of harmonic analysis in superspace with the Howe dual pair $\left(O(m) \times S p(2 n), \mathfrak{s l}_{2}\right)$ has the following problems,

- P1: The weight vectors $R^{2 j} \mathscr{H}_{k}$ of $\mathfrak{s l}_{2}$ are not irreducible $O(m) \times S p(2 n)$ representations.

- P2: The polynomials $R^{2 j}$ are not the only polynomials invariant under $O(m) \times S p(2 n)$.

- P3: The supersphere integration is not uniquely determined by the $O(m) \times S p(2 n)$-invariance and the modulo $R^{2}-1$ property.

These problems will be solved by introducing the orthosymplectic Lie supergroup.

Our approach to Lie supergroups corresponds to the one in [1]. The details of the definitions can be found there. A Lie supergroup is a supermanifold $\mathscr{G}=\left(\mathscr{G}_{0}, \mathscr{O}_{\mathscr{G}}\right)$ equipped with the additional structure of a supermanifold morphism $\mu: \mathscr{G} \times \mathscr{G} \rightarrow \mathscr{G}$ satisfying the associativity property and an involutive superdiffeomorphism $\sigma: \mathscr{G} \rightarrow \mathscr{G}$. There is a distinguished point $e_{\mathscr{G}}$ in the underlying manifold $\mathscr{G}_{0}$, which satisfies the identity property.

The action of a Lie supergroup $\mathscr{G}=\left(\mathscr{G}_{0}, \mathscr{O}_{\mathscr{G}}\right)$ on a supermanifold $\mathscr{M}=\left(\mathscr{M}_{0}, \mathscr{O}_{\mathscr{M}}\right)$ is given by a supermanifold morphism

$$
\Psi:\left(\mathscr{G}_{0}, \mathscr{O}_{\mathscr{G}}\right) \times\left(\mathscr{M}_{0}, \mathscr{O}_{\mathscr{M}}\right) \rightarrow\left(\mathscr{M}_{0}, \mathscr{O}_{\mathscr{M}}\right)
$$

which satisfies the logical conditions. 


\section{INVARIANT FUNCTIONS AND HARISH-CHANDRA PAIRS}

In [1] the Lie superalgebra of a Lie supergroup is defined. The Lie superalgebra $\mathfrak{g}$ corresponding to $\mathscr{G}$ is given by the elements of $\operatorname{Der} \mathscr{O}_{\mathscr{G}}\left(\mathscr{G}_{0}\right)$ which are right (or left) invariant. Here Der $\mathscr{O}_{\mathscr{G}}$ is the sheaf over $\mathscr{G}_{0}$ of superderivations of the structure sheaf $\mathscr{O}_{\mathscr{G}}$. For our purposes it will be more convenient to use a different and stronger characterization compared to [1], it can be proven however that the resulting algebra is identical.

Definition 2. Consider a the Lie supergroup $\mathscr{G}$ with multiplication $\mu$. The Lie superalgebra $\mathfrak{g}$ corresponding to $\mathscr{G}$ is the algebra with multiplication given by the Lie superbracket and with elements given by

$$
\left\{X \in \operatorname{Der} \mathscr{O}_{\mathscr{G}}\left(\mathscr{G}_{0}\right) \mid \mu^{\sharp} \circ X=\left(X \times i d_{\mathscr{G}}^{\sharp}\right) \circ \mu^{\sharp}\right\} .
$$

In order to be able to use Harish-Chandra pairs we also define the action of the Lie algebra on the supermanifold.

Definition 3. The action of an element $X \in \mathfrak{g}$ on an element $f \in \mathscr{O}_{\mathscr{M}}\left(\mathscr{M}_{0}\right)$ is given by $\gamma:\left(\mathfrak{g}, \mathscr{O}_{\mathscr{M}}\left(\mathscr{M}_{0}\right)\right) \rightarrow \mathscr{O}_{\mathscr{M}}\left(\mathscr{M}_{0}\right)$,

$$
\gamma(X, f)=\left(\delta_{e \mathscr{G}}^{\sharp} \circ X \times i d_{\mathscr{M}}^{\sharp}\right) \circ \psi^{\sharp}(f) .
$$

In the bosonic case this definition coincides with the one given by differentiating the action of the Lie group. This definition implies an algebra morphism and can be extended to the universal enveloping algebra.

Theorem 2. The isomorphism of vector spaces $\mathfrak{g} \rightarrow \operatorname{Der}_{\mathscr{O}}\left(\mathscr{M}_{0}\right): X \rightarrow \gamma(X, \cdot)$ is a Lie superalgebra morphism.

Now a superfunction $f \in \mathscr{O} \mathscr{M}\left(\mathscr{M}_{0}\right)$ is $\mathscr{G}$-invariant for the action of a Lie supergroup on the super manifold $\mathscr{M}$ if $\psi^{\sharp}(f)=1 \mathscr{G} \times f$. This invariance can be characterized using the underlying Lie group and the Lie superalgebra.

Theorem 3. Given a superfunction $f \in \mathscr{O}_{\mathscr{M}}\left(\mathscr{M}_{0}\right)$ and the action of a Lie supergroup $\mathscr{G}$ with Lie superalgebra $\mathfrak{g}$ on $\mathscr{M}, \Psi: \mathscr{G} \times \mathscr{M} \rightarrow \mathscr{M}$. The superfunction $f$ is $\mathscr{G}$-invariant if and only if $f$ is $\mathscr{G}_{0}$-invariant and

$$
\gamma(X, f)=0
$$

for all $X \in \mathfrak{g}$.

A Harisch-Chandra pair is a pair $\left(\mathscr{G}_{0}, \mathfrak{g}\right)$, consisting of a Lie group $\mathscr{G}_{0}$ and a Lie superalgebra $\mathfrak{g}=\mathfrak{g}_{0} \oplus \mathfrak{g}_{1}$. The algebra $\mathfrak{g}_{0}$ is the Lie algebra of $\mathscr{G}_{0}$ and the Lie superbracket, restricted to $\mathfrak{g}_{0} \times \mathfrak{g}$ exponentiates to $\mathscr{G}_{0} \times \mathfrak{g}$. There is an equivalency in the sense of categories between Harish-Chandra pairs and Lie supergroups. Theorem 3 implies that the characterization of invariant functions is equivalent in the supermanifold or the Harish-Chandra approach.

The Lie group $O(m)$ is the group of isometries of $\mathbb{R}^{m}$ which stabilize the origin. In [5], the isometry supergroup of a Riemannian supermanifold is defined in terms of its Harish-Chandra pair. Applying the definition to our flat Riemannian superspace yields

Theorem 4. The supergroup of isometries of the Riemannian superspace $\mathbb{R}^{m \mid 2 n}$ which stabilize the origin is the Lie supergroup $\operatorname{OSp}(m \mid 2 n)$, which corresponds to the Harish-Chandra pair

$$
(O(m) \times S p(2 n), \mathfrak{o s p}(m \mid 2 n)),
$$

the action of $\mathfrak{o s p}(m \mid 2 n)$ on $\mathscr{C}^{\infty}\left(\mathbb{R}^{m}\right) \otimes \Lambda_{2 n}$ is given by

$$
L_{i j}=X_{i} \partial_{X^{j}}-(-1)^{[i][j]} X_{j} \partial_{X^{i}}
$$

for $1 \leq i \leq j \leq m+2 n$ and $[i]=0$ if $i \leq m$ and $[i]=1$ otherwise.

Theorem 3 implies that the orthosymplectically invariant functions on $\mathbb{R}^{m \mid 2 n}$ are those which are invariant under $O(m) \times S p(2 n)$ and are null-solutions of all the $L_{i j}$. Therefore the results in [3] imply that the functions of the form

$$
f(\mathbf{x})=h\left(R^{2}\right)=\sum_{j=0}^{n} h^{(j)}\left(r^{2}\right) \frac{\theta^{2 j}}{j !} .
$$

with $h \in \mathscr{C}^{\infty}\left(\mathbb{R}^{+}\right)$are the only orthosymplectically invariant functions, this solves $\mathbf{P 2}$. 


\section{INTEGRATION OVER THE SUPERSPHERE}

In the bosonic case the unit sphere integration $\int_{\mathbb{S}^{m-1}}: \mathscr{C}^{\infty}\left(\mathbb{R}^{m}\right) \rightarrow \mathbb{C}$ is uniquely determined by its $O(m)$-invariance and the fact that $\int_{\mathbb{S}^{m-1}} f=0$ when $f$ vanishes on $\mathbb{S}^{m-1}$. Also the $\operatorname{OSp}(m \mid 2 n)$-invariance of a linear functional can be expressed using the Harish-Chandra pair. Based on equation (4) the logical characterization for $f(\mathbf{x})=0$ for $R=1$ is $\left[\sum_{j=0}^{n} \frac{(-1)^{j} \theta^{2 j}}{j !}\left(\frac{\partial}{\partial r^{2}}\right)^{j} f(\mathbf{x})\right]_{r=1}=0$. The following theorem can then be proven, which solves $\mathbf{P 3}$.

Theorem 5. When $m \neq 0$, the only (up to a multiplicative constant) linear functional $T$ on $\mathscr{O}_{\mathbb{R}^{m} \mid 2 n}^{\infty}\left(\mathbb{R}^{m}\right)$ satisfying the properties

- $T$ is $\operatorname{OSp}(m \mid 2 n)$-invariant

- if $\left[\sum_{j=0}^{n} \frac{(-1)^{j} \theta^{2 j}}{j !}\left(\frac{\partial}{\partial r^{2}}\right)^{j} f(\mathbf{x})\right]_{r=1}=0$, then $T[f]=0$.

is given by

$$
\int_{\mathbb{S}^{m-1 \mid 2 n}} f(\mathbf{x})=\int_{\mathbb{S}^{m-1}} \int_{B}\left(1-\theta^{2}\right)^{\frac{m}{2}-1} \sum_{j=0}^{n} \frac{(-1)^{j} \theta^{2 j}}{j !}\left(\frac{\partial}{\partial r^{2}}\right)^{j} f(\mathbf{x}) .
$$

In [2] it was proven that this expression is equal to the expression in theorem 1 for polynomials. The existence and explicit identity of an orthosymplectically invariant linear functional on the supersphere is important to develop supersymmetric gauge theories on the supersphere.

\section{POLYNOMIALS ON THE SUPERSPHERE}

In [7] it is proven that $\mathscr{H}_{k}$ is an irreducible osp $(m \mid 2 n)$-module for $m-2 n>0$. The following generalization solves P1.

Theorem 6. When $m-2 n \notin-2 \mathbb{N}$, the space $\mathscr{H}_{k}$ is an irreducible osp $(m \mid 2 n)$-module. When $m-2 n \in-2 \mathbb{N}$, $\mathscr{H}_{k}$ is irreducible if and only if $k>2+2 n-m$ or $k<2+n-\frac{m}{2}$. When $2+n-\frac{m}{2} \leq k \leq 2+2 n-m$,

$$
R^{2 k+m-2 n-2} \mathscr{H}_{2+2 n-m-k} \subset \mathscr{H}_{k} .
$$

In the following subsections we present the consequences of this theorem.

\section{The case $m=1$}

For the simple root system of $\mathfrak{o s p}(1 \mid 2 n)$ we choose the standard simple root system of $\mathfrak{s p}(2 n)$. The only non-zero spaces of spherical harmonics are $\mathscr{H}_{k}$ for $k \leq 2 n+1$. As osp $(1 \mid 2 n)$-representations

$$
\mathscr{H}_{k} \cong L_{(1, \cdots, 1,0, \cdots, 0)}^{1 \mid 2 n}
$$

with $L_{(1, \cdots, 1,0 \cdots, 0)}^{1 \mid 2 n}$ the $\mathfrak{o s p}(1 \mid 2 n)$ representation with highest weight $(1, \cdots, 1,0 \cdots, 0)$, where the integer 1 is to be repeated $k$ times when $k \leq n$ and $2 n-k+1$ times when $k>n$. The previous results also determine the representation of supersymmetric tensors of degree $k$ for the natural module $V$ for $\mathfrak{o s p}(m \mid 2 n), S(V)_{k}$. This representation can be identified with $\mathscr{P}_{k}$. The Fischer decomposition (1) using the fact $\mathscr{H}_{k}=\emptyset$ for $k>2 n+1$ implies for $k=2 p$,

$$
S(V)_{2 p} \cong \bigoplus_{j=0}^{\min (n, p)} R^{2 p-2 j} \mathscr{H}_{2 j} \cong \bigoplus_{j=0}^{\min \left(\left\lfloor\frac{n}{2}\right\rfloor, p\right)} L_{\left(\underline{1}_{2 j}, \underline{0}_{n-2 j}\right)}^{1 \mid 2 n} \oplus \bigoplus_{j=\left\lfloor\frac{n}{2}\right\rfloor+1}^{\min (n, p)} L_{\left(\underline{1}_{2 n-2 j+1}, \underline{2}_{2 j-1-n}\right)}^{1 \mid 2 n},
$$

the case $k$ odd is similar. Finally, decomposition (1) also leads to the following conclusion. The space $\mathscr{P}$, as an $\mathfrak{s l}_{2} \times \mathfrak{o s p}(1 \mid 2 n)$-module is given by

$$
\mathscr{P} \cong \sum_{k=0}^{n} L^{(k+1 / 2-n)} \otimes L_{\left(\underline{1}_{k}, \underline{0}_{n-k}\right)}^{1 \mid 2 n} \oplus \sum_{k=n+1}^{2 n+1} L^{(k+1 / 2-n)} \otimes L_{\left(\underline{1}_{2 n-k+1}, \underline{0}_{k-1-n}\right)}^{1 \mid 2 n},
$$

where $L^{(k+1 / 2-n)}$ is the irreducible $\mathfrak{s l}_{2}$-module with lowest weight $k+1 / 2-n$. 


\section{The case $m-2 n \notin-2 \mathbb{N}$ with $m>1$}

The simple root system of $\mathfrak{o s p}(m \mid 2 n)$ as chosen in [7] is used. The space of spherical harmonics, as an osp $(m \mid 2 n)$ representation is $\mathscr{H}_{k} \cong L_{(k, 0, \cdots, 0)}^{m \mid 2 n}$. The representation of supersymmetric tensors of degree $k$ for the natural module $V$ for $\mathfrak{o s p}(m \mid 2 n)$ is

$$
S(V)_{k} \cong \mathscr{P}_{k}=\bigoplus_{j=0}^{\left\lfloor\frac{k}{2}\right\rfloor} R^{2 j} \mathscr{H}_{k-2 j} \cong \bigoplus_{j=0}^{\lfloor k / 2\rfloor} L_{k-2 j, 0, \cdots, 0}^{m \mid 2 n}
$$

Finally, as an $\mathfrak{s l}_{2} \times \mathfrak{o} \mathfrak{s p}(m \mid 2 n)$-module, the space $\mathscr{P}$ is given by $\mathscr{P} \cong \sum_{k=0}^{\infty} L^{(k+m / 2-n)} \otimes L_{(k, 0, \cdots, 0)}^{m \mid 2 n}$.

\section{The case $m-2 n \in-2 \mathbb{N}$}

Also in case $m-2 n \in-2 \mathbb{N}$, the representations still satisfy $\mathscr{H}_{k}=L_{(k, 0, \cdots, 0)}^{m \mid 2 n}$. Contrary to finite dimensional Lie algebras, finite dimensional Lie superalgebras do not possess the complete reducibility property.

Corollary 1. When $m-2 n \in-2 \mathbb{N}$ and $2+n-\frac{m}{2} \leq k \leq 2+2 n-m$ the space $\mathscr{H}_{k}$ is not a completely reducible $\mathfrak{o s p}(m \mid 2 n)$-module.

Clearly, in case $2+n-\frac{m}{2} \leq k \leq 2+2 n-m$, there appears a weight $(2-M-k, 0 \cdots, 0)$ different from $(k, 0, \cdots, 0)$ which is annihilated by all the positive even or odd simple roots. We conclude that

$$
L_{(2+2 n-m-k, 0, \cdots, 0)}^{m \mid 2 n} \subset L_{(k, 0, \cdots, 0)}^{m \mid 2 n}
$$

and $L_{(k, 0, \cdots, 0)}^{m \mid 2 n}$ is an atypical module, see [6].

\section{REFERENCES}

1. C.P. Boyer, and O.A. Sánchez-Valenzuela, Trans. Amer. Math. Soc. 323, no. 1, 151-175. (1991).

2. K. Coulembier, H. De Bie, and F. Sommen, J. Phys. A: Math. Theor. 42, 395206 (2009).

3. K. Coulembier, H. De Bie, and F. Sommen, accepted in J. Math. Phys. (2010).

4. H. De Bie, D. Eelbode, and F. Sommen, J. Phys. A: Math. Theor. 42, 245204 (2009).

5. O. Goertsches, Math. Z. 260, no. 3, 557-593. (2008).

6. V. Kac, Lecture Notes in Math. 676, Springer, Berlin, (1978).

7. R.B. Zhang, Comm. Math. Phys. 280, 545-562 (2008). 